\title{
On a strengthened Hardy-Hilbert type inequality
}

Di-Yi Chen ${ }^{1}$, Guang-Sheng Chen ${ }^{2 *}$, Ti Song ${ }^{3}$ and Li-Fang Liao ${ }^{4}$

\section{"Correspondence:}

cgswavelets@126.com

2Department of Construction and

Information Engineering, Guangxi

Modern Vocational Technology

College, Hechi, Guangxi 547000,

China

Full list of author information is

available at the end of the article

\begin{abstract}
We derive a strengthenment of a Hardy-Hilbert type inequality by using the Euler-Maclaurin expansion for the zeta function and estimating the weight function effectively. As applications, some particular results are presented.
\end{abstract}

MSC: $26 \mathrm{D} 15$

Keywords: Hardy-Hilbert type inequality; weight coefficient; Hölder inequality

\section{Introduction}

Let $p, q>1, \frac{1}{p}+\frac{1}{q}=1, a_{n}, b_{n} \geq 0,0<\sum_{n=1}^{\infty} a_{n}^{p}<\infty$ and $0<\sum_{n=1}^{\infty} b_{n}^{q}<\infty$. Then one [1] has

$$
\begin{aligned}
& \sum_{n=1}^{\infty} \sum_{m=1}^{\infty} \frac{a_{m} b_{n}}{m+n}<\frac{\pi}{\sin (\pi / p)}\left[\sum_{n=1}^{\infty} a_{n}^{p}\right]^{\frac{1}{p}}\left[\sum_{n=1}^{\infty} b_{n}^{q}\right]^{\frac{1}{q}}, \\
& \sum_{n=1}^{\infty} \sum_{m=1}^{\infty} \frac{a_{m} b_{n}}{\max \{m, n\}}<p q\left[\sum_{n=1}^{\infty} a_{n}^{p}\right]^{\frac{1}{p}}\left[\sum_{n=1}^{\infty} b_{n}^{q}\right]^{\frac{1}{q}},
\end{aligned}
$$

where the constant factor $\frac{\pi}{\sin (\pi / p)}$ and $p q$ are best possible. Inequality (1.1) is well known as Hardy-Hilbert's inequality, and inequality (1.2) is named a Hardy-Hilbert type inequality. Both of them are important in analysis and applications [2]. In recent years, many results about generalizations of this type of inequality were established (see [3]). Under the same conditions as (1.1) and (1.2), some Hardy-Hilbert type inequalities, which are similar to (1.1) and (1.2), have been studied and generalized by some mathematicians.

By introducing a parameter, Yang gave a generalization of inequality (1.2) with the best constant factor as follows:

If $p, q>1, \frac{1}{p}+\frac{1}{q}=1,2-\min \{p, q\}<\lambda \leq 2, a_{n}, b_{n} \geq 0$, such that $0<\sum_{n=1}^{\infty} n^{1-\lambda} a_{n}^{p}<\infty$ and $0<\sum_{n=1}^{\infty} n^{1-\lambda} b_{n}^{q}<\infty$, then

$$
\sum_{n=1}^{\infty} \sum_{m=1}^{\infty} \frac{a_{m} b_{n}}{\max \left\{m^{\lambda}, n^{\lambda}\right\}}<k_{\lambda}(p)\left\{\sum_{n=1}^{\infty} n^{1-\lambda} a_{n}^{p}\right\}^{\frac{1}{p}}\left\{\sum_{n=1}^{\infty} n^{1-\lambda} b_{n}^{q}\right\}^{\frac{1}{q}},
$$

where the constant factor $k_{\lambda}(p)=\frac{\lambda p q}{(p+\lambda-2)(q+\lambda-2)}$ is best possible.

Furthermore, by introducing a parameter and two pairs of conjugate exponents, Zhong gave a generalization of inequality (1.3) with the best constant factor as follows:

@ 2013 Chen et al.; licensee Springer. This is an Open Access article distributed under the terms of the Creative Commons Attribution License (http://creativecommons.org/licenses/by/2.0), which permits unrestricted use, distribution, and reproduction in any medium, provided the original work is properly cited. 
If $p>1, \frac{1}{p}+\frac{1}{q}=1, r>1, \frac{1}{r}+\frac{1}{s}=1,0<\lambda \leq \min \{r, s\}, a_{n}, b_{n} \geq 0$, such that $0<$ $\sum_{n=1}^{\infty} n^{p\left(1-\frac{\lambda}{r}\right)-1} a_{n}^{p}<\infty$ and $0<\sum_{n=1}^{\infty} n^{q\left(1-\frac{\lambda}{s}\right)-1} b_{n}^{q}<\infty$, then

$$
\sum_{n=1}^{\infty} \sum_{m=1}^{\infty} \frac{a_{m} b_{n}}{\max \left\{m^{\lambda}, n^{\lambda}\right\}}<k_{\lambda}(r)\left\{\sum_{n=1}^{\infty} n^{p\left(1-\frac{\lambda}{r}\right)-1} a_{n}^{p}\right\}^{\frac{1}{p}}\left\{\sum_{n=1}^{\infty} n^{q\left(1-\frac{\lambda}{s}\right)-1} b_{n}^{q}\right\}^{\frac{1}{q}}
$$

where the constant factor $k_{\lambda}(r)=\frac{r s}{\lambda}$ is best possible.

Recently, in [4], Jiang and Hua established an improvement of inequality (1.3) as follows:

If $p, q>1, \frac{1}{p}+\frac{1}{q}=1,2-\min \{p, q\}<\lambda \leq 2, a_{n} \geq 0, b_{n} \geq 0$, for $n \geq 1, n \in \mathrm{N}$ and $0<$ $\sum_{n=1}^{\infty} n^{1-\lambda} a_{n}^{p}<\infty, 0<\sum_{n=1}^{\infty} n^{1-\lambda} b_{n}^{q}<\infty$, then

$$
\begin{aligned}
\sum_{n=1}^{\infty} \sum_{m=1}^{\infty} \frac{a_{m} b_{n}}{\max \left\{m^{\lambda}, n^{\lambda}\right\}}< & \left\{\sum_{n=1}^{\infty}\left[k(\lambda)-\frac{q}{3(q+\lambda-2) n^{\frac{q+\lambda-2}{q}}}\right] n^{1-\lambda} a_{n}^{p}\right\}^{\frac{1}{p}} \\
& \times\left\{\sum_{n=1}^{\infty}\left[k(\lambda)-\frac{p}{3(p+\lambda-2) n^{\frac{p+\lambda-2}{p}}}\right] n^{1-\lambda} b_{n}^{q}\right\},
\end{aligned}
$$

where $k(\lambda)=\frac{p q \lambda}{(p+\lambda-2)(q+\lambda-2)}>0$.

In this paper, by introducing a parameter and estimating the weight coefficient, we obtain a strengthenment of inequality (1.4) and generalize inequality (1.5). As applications, some particular results are presented.

\section{Some preliminary results}

First, we need the following formula of the Riemann- $\zeta$ function (see [5]):

$$
\begin{aligned}
\zeta(\rho)= & \sum_{n=1}^{m} \frac{1}{n^{\rho}}-\frac{m^{1-\rho}}{1-\rho}-\frac{1}{2 m^{\rho}} \\
& -\sum_{n=1}^{l-1} \frac{B_{2 n}}{2 n}\left(\begin{array}{c}
-\rho \\
2 n-1
\end{array}\right) \frac{1}{m^{\rho+2 n-1}}-\frac{B_{2 l}}{2 l}\left(\begin{array}{c}
-\rho \\
2 l-1
\end{array}\right) \frac{\varepsilon}{m^{\rho+2 l-1}},
\end{aligned}
$$

where $\rho>0, \rho \neq 1, m, l \geq 1, m, l \in \mathrm{N}, 0<\varepsilon=\varepsilon(\rho, l, m)<1$. The numbers $B_{1}=-1 / 2, B_{2}=1 / 6$, $B_{3}=0, B_{4}=-1 / 30, \ldots$ are Bernoulli numbers. In particular, $\zeta(\rho)=\sum_{n=1}^{\infty} \frac{1}{n^{\rho}}(\rho>1)$.

Since $\zeta(0)=-1 / 2$, the formula of the Riemann- $\zeta$ function (2.1) also holds for $\rho=0$.

Lemma 2.1 Let $r>1, \frac{1}{r}+\frac{1}{s}=1,0<\lambda \leq \min \{r, s\}$, define the weight coefficients $\omega(m, \lambda, s)$ and $\omega(n, \lambda, r)$ as

$$
\begin{aligned}
& \omega(m, \lambda, s)=\sum_{n=1}^{\infty} \frac{1}{\max \left\{m^{\lambda}, n^{\lambda}\right\}}\left(\frac{m}{n}\right)^{1-\frac{\lambda}{s}}, \\
& \omega(n, \lambda, r)=\sum_{m=1}^{\infty} \frac{1}{\max \left\{m^{\lambda}, n^{\lambda}\right\}}\left(\frac{n}{m}\right)^{1-\frac{\lambda}{r}} .
\end{aligned}
$$

Then we have

$$
\omega(m, \lambda, s)<m^{1-\lambda}\left[k_{\lambda}-\frac{s}{3 \lambda m^{\frac{\lambda}{s}}}\right]
$$


Chen et al. Journal of Inequalities and Applications 2013, 2013:511

Page 3 of 9

http://www.journalofinequalitiesandapplications.com/content/2013/1/511

and

$$
\omega(n, \lambda, r)<n^{1-\lambda}\left[k_{\lambda}-\frac{r}{3 \lambda m^{\frac{\lambda}{r}}}\right]
$$

where $k_{\lambda}=\frac{r s}{\lambda}$.

Proof For $0<\lambda \leq \min \{r, s\}$, taking $\rho=1-\frac{\lambda}{s} \geq 0, l=1$ in (2.1), we get

$$
\zeta\left(1-\frac{\lambda}{s}\right)=\sum_{n=1}^{m} \frac{1}{n^{1-\frac{\lambda}{s}}}-\frac{s m^{\frac{\lambda}{s}}}{\lambda}-\frac{1}{2 m^{1-\frac{\lambda}{s}}}+\frac{1-\frac{\lambda}{s}}{12 m^{2-\frac{\lambda}{s}}} \varepsilon_{1},
$$

where $0<\varepsilon_{1}<1$.

Set $\rho=1+\frac{\lambda}{r}, l=1$, and we can derive

$$
\zeta\left(1+\frac{\lambda}{r}\right)=\sum_{n=1}^{m-1} \frac{1}{n^{1+\frac{\lambda}{r}}}+\frac{r m^{-\frac{\lambda}{r}}}{\lambda}+\frac{1}{2 m^{1+\frac{\lambda}{r}}}+\frac{1+\frac{\lambda}{r}}{12 m^{2+\frac{\lambda}{r}}} \varepsilon_{2},
$$

where $0<\varepsilon_{2}<1$.

Thus we get

$$
\begin{aligned}
\omega(m, \lambda, s) & =\sum_{n=1}^{\infty} \frac{1}{\max \left\{m^{\lambda}, n^{\lambda}\right\}}\left(\frac{m}{n}\right)^{1-\frac{\lambda}{s}} \\
& =\sum_{n=1}^{m} \frac{1}{\max \left\{m^{\lambda}, n^{\lambda}\right\}}\left(\frac{m}{n}\right)^{1-\frac{\lambda}{s}}-\frac{1}{m^{\lambda}}+\sum_{n=m}^{\infty} \frac{1}{\max \left\{m^{\lambda}, n^{\lambda}\right\}}\left(\frac{m}{n}\right)^{1-\frac{\lambda}{s}} \\
& =\sum_{n=1}^{m} \frac{1}{m^{\lambda}}\left(\frac{m}{n}\right)^{1-\frac{\lambda}{s}}-\frac{1}{m^{\lambda}}+\sum_{n=m}^{\infty} \frac{1}{n^{\lambda}}\left(\frac{m}{n}\right)^{1-\frac{\lambda}{s}} \\
& =\frac{1}{m^{\lambda+\frac{\lambda}{s}-1}} \sum_{n=1}^{m} \frac{1}{n^{1-\frac{\lambda}{s}}}-\frac{1}{m^{\lambda}}+m^{1-\frac{\lambda}{s}} \sum_{n=m}^{\infty} \frac{1}{n^{1+\frac{\lambda}{r}}} .
\end{aligned}
$$

Combining (2.6) and (2.7), we have

$$
\begin{aligned}
\omega(m, \lambda, s)< & \frac{1}{m^{\lambda+\frac{\lambda}{s}-1}}\left[\zeta\left(1-\frac{\lambda}{s}\right)+\frac{s m^{\frac{\lambda}{s}}}{\lambda}+\frac{1}{2 m^{1-\frac{\lambda}{s}}}\right]-\frac{1}{m^{\lambda}} \\
& +m^{1-\frac{\lambda}{s}}\left[\frac{r m^{-\frac{\lambda}{r}}}{\lambda}+\frac{1}{2 m^{1+\frac{\lambda}{r}}}+\frac{1+\frac{\lambda}{r}}{12 m^{2+\frac{\lambda}{r}}}\right] \\
= & \frac{1}{m^{\lambda+\frac{\lambda}{s}-1}} \zeta\left(1-\frac{\lambda}{s}\right)+\frac{s m^{1-\lambda}}{\lambda}+\frac{1}{2 m^{\lambda}}-\frac{1}{m^{\lambda}}+\frac{r m^{1-\lambda}}{\lambda}+\frac{1}{2 m^{\lambda}}+\frac{1+\frac{\lambda}{r}}{12 m^{1+\lambda}} \\
= & \frac{1}{m^{\lambda+\frac{\lambda}{s}-1}} \zeta\left(1-\frac{\lambda}{s}\right)+\frac{r s m^{1-\lambda}}{\lambda}+\frac{1+\frac{\lambda}{r}}{12 m^{1+\lambda}} \\
= & m^{1-\lambda}\left\{\frac{r s}{\lambda}-\frac{1}{m^{\frac{\lambda}{s}}}\left[-\zeta\left(1-\frac{\lambda}{s}\right)-\frac{1+\frac{\lambda}{r}}{12 m^{2-\frac{\lambda}{s}}}\right]\right\} .
\end{aligned}
$$


In (2.6), let $m=1$, by $0<\lambda \leq \min \{r, s\}$, we obtain

$$
\begin{aligned}
\zeta\left(1-\frac{\lambda}{s}\right) & =1-\frac{s}{\lambda}-\frac{1}{2}+\frac{\left(1-\frac{\lambda}{s}\right) \varepsilon_{1}}{12}<\frac{1}{2}-\frac{s}{\lambda}+\frac{1-\frac{\lambda}{s}}{12}=\frac{6 \lambda-12 s-\lambda\left(1-\frac{\lambda}{s}\right)}{12 \lambda} \\
& <\frac{6 \lambda-12 s-(\lambda-s)}{12 \lambda}=\frac{5 \lambda-11 s}{12 \lambda}=-\frac{11 s-5 \lambda}{12 \lambda}<0 .
\end{aligned}
$$

Therefore, for $m \geq 1, m \in \mathrm{N}, 0<\lambda \leq \min \{r, s\}$, we obtain

$$
\begin{aligned}
-\zeta\left(1-\frac{\lambda}{s}\right)-\frac{1+\frac{\lambda}{r}}{12 m^{2-\frac{\lambda}{s}}} & >\frac{11 s-5 \lambda}{12 \lambda}-\frac{1+\frac{\lambda}{r}}{12}=\frac{11 s-5 \lambda-\lambda\left(1+\frac{\lambda}{r}\right)}{12 \lambda} \geq \frac{11 s-5 \lambda-2 \lambda}{12 \lambda} \\
& =\frac{4 s+7(s-\lambda)}{12 \lambda} \geq \frac{4 s}{12 \lambda}=\frac{s}{3 \lambda} .
\end{aligned}
$$

Applying the above inequality, we obtain (2.4). Similarly, we can prove (2.5). The lemma is proved.

\section{Main results}

Theorem 3.1 Assume that $p, q>1, \frac{1}{p}+\frac{1}{q}=1, r>1, \frac{1}{r}+\frac{1}{s}=1,0<\lambda \leq \min \{r, s\}, a_{n} \geq 0$, $b_{n} \geq 0$, such that $0<\sum_{n=1}^{\infty} n^{p\left(1-\frac{\lambda}{r}\right)-1} a_{n}^{p}<\infty$ and $0<\sum_{n=1}^{\infty} n^{q\left(1-\frac{\lambda}{s}\right)-1} b_{n}^{q}<\infty$, then

$$
\begin{aligned}
& \sum_{n=1}^{\infty} \sum_{m=1}^{\infty} \frac{a_{m} b_{n}}{\max \left\{m^{\lambda}, n^{\lambda}\right\}}<\left\{\sum_{n=1}^{\infty}\left[k_{\lambda}-\frac{s}{3 \lambda n^{\frac{\lambda}{s}}}\right] n^{p\left(1-\frac{\lambda}{r}\right)-1} a_{n}^{p}\right\}^{\frac{1}{p}} \\
& \times\left\{\sum_{n=1}^{\infty}\left[k_{\lambda}-\frac{r}{3 \lambda n^{\frac{\lambda}{r}}}\right] n^{q\left(1-\frac{\lambda}{s}\right)-1} b_{n}^{q}\right\}^{\frac{1}{q}}, \\
& \sum_{n=1}^{\infty} \frac{n^{\frac{p \lambda}{s}}-1}{\left[k_{\lambda}-\frac{r}{3 \lambda n^{\frac{\lambda}{r}}}\right]^{p-1}}\left[\sum_{m=1}^{\infty} \frac{a_{m}}{\max \left\{m^{\lambda}, n^{\lambda}\right\}}\right]^{p}<\sum_{n=1}^{\infty}\left[k_{\lambda}-\frac{s}{3 \lambda n^{\frac{\lambda}{s}}}\right] n^{p\left(1-\frac{\lambda}{r}\right)-1} a_{n}^{p},
\end{aligned}
$$

where $k_{\lambda}=\frac{r s}{\lambda}>0$. Inequality (3.1) is equivalent to (3.2). In particular, we have the following equivalent inequalities:

$$
\begin{aligned}
\sum_{n=1}^{\infty} \sum_{m=1}^{\infty} \frac{a_{m} b_{n}}{\max \left\{m^{\lambda}, n^{\lambda}\right\}}< & k_{\lambda}\left\{\sum_{n=1}^{\infty}\left[1-\frac{s}{k_{\lambda} 3 \lambda n^{\frac{\lambda}{s}}}\right] n^{p\left(1-\frac{\lambda}{r}\right)-1} a_{n}^{p}\right\}^{\frac{1}{p}} \\
& \times\left\{\sum_{n=1}^{\infty} n^{q\left(1-\frac{\lambda}{s}\right)-1} b_{n}^{q}\right\}^{\frac{1}{q}}, \\
\sum_{n=1}^{\infty} n^{\frac{p \lambda}{s}}-1 & {\left[\sum_{m=1}^{\infty} \frac{a_{m}}{\max \left\{m^{\lambda}, n^{\lambda}\right\}}\right]^{p}<k_{\lambda}^{p} \sum_{n=1}^{\infty}\left[1-\frac{s}{3 k_{\lambda} \lambda n^{\frac{\lambda}{s}}}\right] n^{p\left(1-\frac{\lambda}{r}\right)-1} a_{n}^{p} . }
\end{aligned}
$$

Proof From Hölder inequality (see [6]), we have

$$
\begin{aligned}
& \sum_{n=1}^{\infty} \sum_{m=1}^{\infty} \frac{a_{m} b_{n}}{\max \left\{m^{\lambda}, n^{\lambda}\right\}} \\
& \quad=\sum_{n=1}^{\infty} \sum_{m=1}^{\infty} \frac{a_{m} b_{n}}{\max \left\{m^{\lambda}, n^{\lambda}\right\}} \frac{n^{(\lambda / s-1) / p}}{m^{(\lambda / r-1) / q}} \frac{m^{(\lambda / r-1) / q}}{n^{(\lambda / s-1) / p}}
\end{aligned}
$$




$$
\begin{aligned}
& \leq\left\{\sum_{n=1}^{\infty} \sum_{m=1}^{\infty} \frac{a_{m}^{p} m^{p(1-\lambda / r)+\lambda-2}}{\max \left\{m^{\lambda}, n^{\lambda}\right\}}\left(\frac{m}{n}\right)^{1-\frac{\lambda}{s}}\right\}^{\frac{1}{p}}\left\{\sum_{n=1}^{\infty} \sum_{m=1}^{\infty} \frac{b_{n}^{q} n^{q(1-\lambda / s)+\lambda-2}}{\max \left\{m^{\lambda}, n^{\lambda}\right\}}\left(\frac{n}{m}\right)^{1-\frac{\lambda}{r}}\right\}^{\frac{1}{q}} \\
& =\left\{\sum_{m=1}^{\infty} \omega(m, \lambda, s) m^{p(1-\lambda / r)+\lambda-2} a_{m}^{p}\right\}^{\frac{1}{p}}\left\{\sum_{n=1}^{\infty} \omega(n, \lambda, r) n^{q(1-\lambda / s)+\lambda-2} b_{n}^{q}\right\}^{\frac{1}{q}} .
\end{aligned}
$$

Hence, by (2.4), (2.5), inequality (3.1) is true.

Setting $b_{n}$ as

$$
b_{n}=\frac{n^{p \lambda / s-1}}{\left[k_{\lambda}-\frac{r}{3 \lambda n^{\frac{\lambda}{r}}}\right]^{p-1}}\left[\sum_{m=1}^{\infty} \frac{a_{m}}{\max \left\{m^{\lambda}, n^{\lambda}\right\}}\right]^{p-1}
$$

by using (3.1), we have

$$
\begin{aligned}
\sum_{n=1}^{\infty} & {\left[k_{\lambda}-\frac{r}{3 \lambda n^{\frac{\lambda}{r}}}\right] n^{q\left(1-\frac{\lambda}{s}\right)-1} b_{n}^{q} } \\
= & \sum_{n=1}^{\infty} \frac{n^{p \lambda / s-1}}{\left[k_{\lambda}-\frac{r}{3 \lambda n^{\frac{\lambda}{r}}}\right]^{p-1}}\left[\sum_{m=1}^{\infty} \frac{a_{m}}{\max \left\{m^{\lambda}, n^{\lambda}\right\}}\right]^{p} \\
= & \sum_{n=1}^{\infty} \sum_{m=1}^{\infty} \frac{a_{m} b_{n}}{\max \left\{m^{\lambda}, n^{\lambda}\right\}} \leq\left\{\sum_{n=1}^{\infty}\left[k_{\lambda}-\frac{s}{3 \lambda n^{\frac{\lambda}{s}}}\right] n^{p\left(1-\frac{\lambda}{r}\right)-1} a_{n}^{p}\right\}^{\frac{1}{p}} \\
& \times\left\{\sum_{n=1}^{\infty}\left[k_{\lambda}-\frac{r}{3 \lambda n^{\frac{\lambda}{r}}}\right] n^{q\left(1-\frac{\lambda}{s}\right)-1} b_{n}^{q}\right\}^{\frac{1}{q}} .
\end{aligned}
$$

Hence, we obtain

$$
\begin{aligned}
0 & <\sum_{n=1}^{\infty} \frac{n^{\frac{p \lambda}{s}-1}}{\left[k_{\lambda}-\frac{r}{3 \lambda n^{\frac{\lambda}{r}}}\right]^{p-1}}\left[\sum_{m=1}^{\infty} \frac{a_{m}}{\max \left\{m^{\lambda}, n^{\lambda}\right\}}\right]^{p} \\
& <\sum_{n=1}^{\infty}\left[k_{\lambda}-\frac{s}{3 \lambda n^{\frac{\lambda}{s}}}\right] n^{p\left(1-\frac{\lambda}{r}\right)-1} a_{n}^{p}<\infty .
\end{aligned}
$$

By (3.1), both (3.5) and (3.6) take the form of strict inequality, and we have (3.2).

On the other hand, suppose that (3.2) is valid, from Hölder inequality, we find

$$
\begin{aligned}
& \sum_{n=1}^{\infty} \sum_{m=1}^{\infty} \frac{a_{m} b_{n}}{\max \left\{m^{\lambda}, n^{\lambda}\right\}} \\
& \quad=\sum_{n=1}^{\infty} \frac{n^{[q(\lambda / s-1)+1] / q}}{\left[k_{\lambda}-\frac{r}{3 \lambda n^{\frac{\lambda}{r}}}\right]^{\frac{1}{q}}}\left[\sum_{m=1}^{\infty} \frac{a_{m}}{\max \left\{m^{\lambda}, n^{\lambda}\right\}}\right]\left[k_{\lambda}-\frac{r}{3 \lambda n^{\frac{\lambda}{r}}}\right]^{\frac{1}{q}} n^{[q(1-\lambda / s)-1] / q} b_{n} \\
& \quad \leq\left\{\sum_{n=1}^{\infty} \frac{n^{p \lambda / s-1}}{\left[k_{\lambda}-\frac{r}{3 \lambda n^{\frac{\lambda}{r}}}\right]^{p-1}}\left[\sum_{m=1}^{\infty} \frac{a_{m}}{\max \left\{m^{\lambda}, n^{\lambda}\right\}}\right]^{p}\right\}^{\frac{1}{p}}\left\{\sum_{n=1}^{\infty}\left[k_{\lambda}-\frac{r}{3 \lambda n^{\frac{\lambda}{r}}}\right] n^{q(1-\lambda / s)-1} b_{n}^{q}\right\}^{\frac{1}{q}} .
\end{aligned}
$$

Then, by using (3.2), we have (3.1). Hence, (3.2) and (3.1) are equivalent. The proof of Theorem 3.1 is completed. 
Since $0<\lambda \leq \min \{r, s\}$, by Theorem 3.1, we have the following.

Corollary 3.2 Assume that $p, q>1, \frac{1}{p}+\frac{1}{q}=1, r>1, \frac{1}{r}+\frac{1}{s}=1,0<\lambda \leq \min \{r, s\}, a_{n} \geq 0$, $b_{n} \geq 0$, such that $0<\sum_{n=1}^{\infty} n^{p\left(1-\frac{\lambda}{r}\right)-1} a_{n}^{p}<\infty$ and $0<\sum_{n=1}^{\infty} n^{q\left(1-\frac{\lambda}{s}\right)-1} b_{n}^{q}<\infty$, then

$$
\begin{aligned}
& \sum_{n=1}^{\infty} \sum_{m=1}^{\infty} \frac{a_{m} b_{n}}{\max \left\{m^{\lambda}, n^{\lambda}\right\}}<\left\{\sum_{n=1}^{\infty}\left[k_{\lambda}-\frac{1}{3 n^{\frac{\lambda}{s}}}\right] n^{p\left(1-\frac{\lambda}{r}\right)-1} a_{n}^{p}\right\}^{\frac{1}{p}} \\
& \times\left\{\sum_{n=1}^{\infty}\left[k_{\lambda}-\frac{1}{3 n^{\frac{\lambda}{r}}}\right] n^{q\left(1-\frac{\lambda}{s}\right)-1} b_{n}^{q}\right\}^{\frac{1}{q}}, \\
& \sum_{n=1}^{\infty} \frac{n^{\frac{p \lambda}{s}}-1}{\left[k_{\lambda}-\frac{1}{3 n^{\frac{\lambda}{r}}}\right]^{p-1}}\left[\sum_{m=1}^{\infty} \frac{a_{m}}{\max \left\{m^{\lambda}, n^{\lambda}\right\}}\right]^{p}<\sum_{n=1}^{\infty}\left[k_{\lambda}-\frac{1}{3 n^{\frac{\lambda}{s}}}\right] n^{p\left(1-\frac{\lambda}{r}\right)-1} a_{n}^{p},
\end{aligned}
$$

where $k_{\lambda}=\frac{r s}{\lambda}>0$. Inequality (3.7) is equivalent to (3.8).

For $r=s=2$, by using (3.1) and (3.2), we have the following.

Corollary 3.3 Assume that $p, q>1, \frac{1}{p}+\frac{1}{q}=1,0<\lambda \leq 2, a_{n} \geq 0, b_{n} \geq 0$, such that $0<$ $\sum_{n=1}^{\infty} n^{p\left(1-\frac{\lambda}{2}\right)-1} a_{n}^{p}<\infty$ and $0<\sum_{n=1}^{\infty} n^{q\left(1-\frac{\lambda}{2}\right)-1} b_{n}^{q}<\infty$, then

$$
\begin{aligned}
& \sum_{n=1}^{\infty} \sum_{m=1}^{\infty} \frac{a_{m} b_{n}}{\max \left\{m^{\lambda}, n^{\lambda}\right\}}<\left\{\sum_{n=1}^{\infty}\left[k_{\lambda}-\frac{2}{3 \lambda n^{\frac{\lambda}{2}}}\right] n^{p\left(1-\frac{\lambda}{2}\right)-1} a_{n}^{p}\right\}^{\frac{1}{p}} \\
& \times\left\{\sum_{n=1}^{\infty}\left[k_{\lambda}-\frac{2}{3 \lambda n^{\frac{\lambda}{2}}}\right] n^{q\left(1-\frac{\lambda}{2}\right)-1} b_{n}^{q}\right\}^{\frac{1}{q}}, \\
& \sum_{n=1}^{\infty} \frac{n^{\frac{p \lambda}{2}-1}}{\left[k_{\lambda}-\frac{2}{3 \lambda n^{\frac{\lambda}{2}}}\right]^{p-1}}\left[\sum_{m=1}^{\infty} \frac{a_{m}}{\max \left\{m^{\lambda}, n^{\lambda}\right\}}\right]^{p}<\sum_{n=1}^{\infty}\left[k_{\lambda}-\frac{2}{3 \lambda n^{\frac{\lambda}{2}}}\right] n^{p\left(1-\frac{\lambda}{2}\right)-1} a_{n}^{p},
\end{aligned}
$$

where $k_{\lambda}=\frac{4}{\lambda}>0$. Inequality (3.9) is equivalent to (3.10). In particular, we have the equivalent inequalities as follows.

$$
\begin{aligned}
\sum_{n=1}^{\infty} \sum_{m=1}^{\infty} \frac{a_{m} b_{n}}{\max \left\{m^{\lambda}, n^{\lambda}\right\}}< & k_{\lambda}\left\{\sum_{n=1}^{\infty}\left[1-\frac{2}{k_{\lambda} 3 \lambda n^{\frac{\lambda}{2}}}\right] n^{p\left(1-\frac{\lambda}{2}\right)-1} a_{n}^{p}\right\}^{\frac{1}{p}} \\
& \times\left\{\sum_{n=1}^{\infty} n^{q\left(1-\frac{\lambda}{2}\right)-1} b_{n}^{q}\right\}^{\frac{1}{q}}, \\
\sum_{n=1}^{\infty} n^{\frac{p \lambda}{2}}-1 & {\left[\sum_{m=1}^{\infty} \frac{a_{m}}{\max \left\{m^{\lambda}, n^{\lambda}\right\}}\right]^{p}<k_{\lambda}^{p} \sum_{n=1}^{\infty}\left[1-\frac{2}{3 k_{\lambda} \lambda n^{\frac{\lambda}{2}}}\right] n^{p\left(1-\frac{\lambda}{2}\right)-1} a_{n}^{p} . }
\end{aligned}
$$

For $r=q, s=p$, by using (3.1) and (3.2), we have the following. 
Corollary 3.4 Assume that $p, q>1, \frac{1}{p}+\frac{1}{q}=1,0<\lambda \leq \min \{p, q\}, a_{n} \geq 0, b_{n} \geq 0$, such that $0<\sum_{n=1}^{\infty} n^{(p-1)(1-\lambda)} a_{n}^{p}<\infty$ and $0<\sum_{n=1}^{\infty} n^{(q-1)(1-\lambda)} b_{n}^{q}<\infty$, then

$$
\begin{aligned}
& \sum_{n=1}^{\infty} \sum_{m=1}^{\infty} \frac{a_{m} b_{n}}{\max \left\{m^{\lambda}, n^{\lambda}\right\}}<\left\{\sum_{n=1}^{\infty}\left[k_{\lambda}-\frac{p}{3 \lambda n^{\frac{\lambda}{p}}}\right] n^{(p-1)(1-\lambda)} a_{n}^{p}\right\}^{\frac{1}{p}} \\
& \times\left\{\sum_{n=1}^{\infty}\left[k_{\lambda}-\frac{q}{3 \lambda n^{\frac{\lambda}{q}}}\right] n^{(q-1)(1-\lambda)} b_{n}^{q}\right\}^{\frac{1}{q}}, \\
& \sum_{n=1}^{\infty} \frac{n^{\lambda-1}}{\left[k_{\lambda}-\frac{q}{3 \lambda n^{\frac{\lambda}{q}}}\right]^{p-1}}\left[\sum_{m=1}^{\infty} \frac{a_{m}}{\max \left\{m^{\lambda}, n^{\lambda}\right\}}\right]^{p}<\sum_{n=1}^{\infty}\left[k_{\lambda}-\frac{p}{3 \lambda n^{\frac{\lambda}{p}}}\right] n^{(p-1)(1-\lambda)} a_{n}^{p},
\end{aligned}
$$

where $k_{\lambda}=\frac{p q}{\lambda}>0$. Inequality (3.13) is equivalent to (3.14). In particular, we have the equivalent inequalities as follows.

$$
\begin{gathered}
\sum_{n=1}^{\infty} \sum_{m=1}^{\infty} \frac{a_{m} b_{n}}{\max \left\{m^{\lambda}, n^{\lambda}\right\}}<k_{\lambda}\left\{\sum_{n=1}^{\infty}\left[1-\frac{p}{k_{\lambda} 3 \lambda n^{\frac{\lambda}{p}}}\right] n^{(p-1)(1-\lambda)} a_{n}^{p}\right\}^{\frac{1}{p}} \\
\times\left\{\sum_{n=1}^{\infty} n^{(q-1)(1-\lambda)} b_{n}^{q}\right\}^{\frac{1}{q}}, \\
\sum_{n=1}^{\infty} n^{\lambda-1}\left[\sum_{m=1}^{\infty} \frac{a_{m}}{\max \left\{m^{\lambda}, n^{\lambda}\right\}}\right]^{p}<k_{\lambda}^{p} \sum_{n=1}^{\infty}\left[1-\frac{p}{3 k_{\lambda} \lambda n^{\frac{\lambda}{p}}}\right] n^{(p-1)(1-\lambda)} a_{n}^{p} .
\end{gathered}
$$

For $r=p, s=q$, by using (3.1) and (3.2), we have the following.

Corollary 3.5 Assume that $p, q>1, \frac{1}{p}+\frac{1}{q}=1,0<\lambda \leq \min \{p, q\}, a_{n} \geq 0, b_{n} \geq 0$, such that $0<\sum_{n=1}^{\infty} n^{p-\lambda-1} a_{n}^{p}<\infty$ and $0<\sum_{n=1}^{\infty} n^{q-\lambda-1} b_{n}^{q}<\infty$, then

$$
\begin{aligned}
& \sum_{n=1}^{\infty} \sum_{m=1}^{\infty} \frac{a_{m} b_{n}}{\max \left\{m^{\lambda}, n^{\lambda}\right\}}<\left\{\sum_{n=1}^{\infty}\left[k_{\lambda}-\frac{q}{3 \lambda n^{\frac{\lambda}{q}}}\right] n^{p-\lambda-1} a_{n}^{p}\right\}^{\frac{1}{p}} \\
& \times\left\{\sum_{n=1}^{\infty}\left[k_{\lambda}-\frac{p}{3 \lambda n^{\frac{\lambda}{p}}}\right] n^{q-\lambda-1} b_{n}^{q}\right\}^{\frac{1}{q}}, \\
& \sum_{n=1}^{\infty} \frac{n^{(p-1) \lambda-1}}{\left[k_{\lambda}-\frac{p}{3 \lambda n^{\frac{\lambda}{p}}}\right]^{p-1}}\left[\sum_{m=1}^{\infty} \frac{a_{m}}{\max \left\{m^{\lambda}, n^{\lambda}\right\}}\right]^{p}<\sum_{n=1}^{\infty}\left[k_{\lambda}-\frac{q}{3 \lambda n^{\frac{\lambda}{q}}}\right] n^{p-\lambda-1} a_{n}^{p},
\end{aligned}
$$

where $k_{\lambda}=\frac{p q}{\lambda}>0$. Inequality (3.17) is equivalent to (3.18). In particular, we have the equivalent inequalities as follows.

$$
\begin{aligned}
& \sum_{n=1}^{\infty} \sum_{m=1}^{\infty} \frac{a_{m} b_{n}}{\max \left\{m^{\lambda}, n^{\lambda}\right\}}<k_{\lambda}\left\{\sum_{n=1}^{\infty}\left[1-\frac{q}{k_{\lambda} 3 \lambda n^{\frac{\lambda}{q}}}\right] n^{p-\lambda-1} a_{n}^{p}\right\}^{\frac{1}{p}}\left\{\sum_{n=1}^{\infty} n^{q-\lambda-1} b_{n}^{q}\right\}^{\frac{1}{q}}, \\
& \sum_{n=1}^{\infty} n^{(p-1) \lambda-1}\left[\sum_{m=1}^{\infty} \frac{a_{m}}{\max \left\{m^{\lambda}, n^{\lambda}\right\}}\right]^{p}<k_{\lambda}^{p} \sum_{n=1}^{\infty}\left[1-\frac{q}{3 k_{\lambda} \lambda n^{\frac{\lambda}{q}}}\right] n^{p-\lambda-1} a_{n}^{p} .
\end{aligned}
$$


Set $\lambda=1$, combining (3.1) and (3.2), we have the following.

Corollary 3.6 Assume that $p, q>1, \frac{1}{p}+\frac{1}{q}=1, r>1, \frac{1}{r}+\frac{1}{s}=1, a_{n} \geq 0, b_{n} \geq 0$, such that $0<\sum_{n=1}^{\infty} n^{\frac{p}{s}-1} a_{n}^{p}<\infty$ and $0<\sum_{n=1}^{\infty} n^{\frac{q}{r}-1} b_{n}^{q}<\infty$, then

$$
\begin{aligned}
& \sum_{n=1}^{\infty} \sum_{m=1}^{\infty} \frac{a_{m} b_{n}}{\max \{m, n\}}<\left\{\sum_{n=1}^{\infty}\left[r s-\frac{s}{3 n^{\frac{1}{s}}}\right] n^{\frac{p}{s}-1} a_{n}^{p}\right\}^{\frac{1}{p}} \\
& \times\left\{\sum_{n=1}^{\infty}\left[r s-\frac{r}{3 n^{\frac{1}{r}}}\right]^{\frac{q}{r}-1} b_{n}^{q}\right\}^{\frac{1}{q}}, \\
& \sum_{n=1}^{\infty} \frac{n^{\frac{p}{s}-1}}{\left[r s-\frac{r}{3^{\frac{1}{r}}}\right]^{p-1}}\left[\sum_{m=1}^{\infty} \frac{a_{m}}{\max \{m, n\}}\right]^{p}<\sum_{n=1}^{\infty}\left[r s-\frac{s}{3 n^{\frac{1}{s}}}\right] n^{\frac{p}{s}-1} a_{n}^{p} .
\end{aligned}
$$

In particular, we have the equivalent inequalities as follows.

$$
\begin{aligned}
& \sum_{n=1}^{\infty} \sum_{m=1}^{\infty} \frac{a_{m} b_{n}}{\max \{m, n\}}<r s\left\{\sum_{n=1}^{\infty}\left[1-\frac{1}{3 r n^{\frac{1}{s}}}\right] n^{\frac{p}{s}-1} a_{n}^{p}\right\}\left\{\sum_{n=1}^{\frac{1}{p}} n^{\frac{q}{r}-1} b_{n}^{q}\right\}^{\frac{1}{q}}, \\
& \sum_{n=1}^{\infty} n^{\frac{p}{s}-1}\left[\sum_{m=1}^{\infty} \frac{a_{m}}{\max \{m, n\}}\right]^{p}<(r s)^{p} \sum_{n=1}^{\infty}\left[1-\frac{1}{3 r n^{\frac{1}{s}}}\right] n^{\frac{p}{s}-1} a_{n}^{p} .
\end{aligned}
$$

Taking $p=q=r=s=2$, in (3.23) and (3.24), we have

$$
\begin{aligned}
& \sum_{n=1}^{\infty} \sum_{m=1}^{\infty} \frac{a_{m} b_{n}}{\max \{m, n\}}<4\left\{\sum_{n=1}^{\infty}\left[1-\frac{1}{6 \sqrt{n}}\right] a_{n}^{2}\right\}^{\frac{1}{2}}\left\{\sum_{n=1}^{\infty}\left[1-\frac{1}{6 \sqrt{n}}\right] b_{n}^{2}\right\}^{\frac{1}{2}}, \\
& \sum_{n=1}^{\infty}\left[\sum_{m=1}^{\infty} \frac{a_{m}}{\max \{m, n\}}\right]^{2}<16 \sum_{n=1}^{\infty}\left[1-\frac{1}{6 \sqrt{n}}\right] a_{n}^{2} .
\end{aligned}
$$

Remark 3.1 For $r=\frac{\lambda p}{\lambda+p-2}$ and $s=\frac{\lambda q}{\lambda+q-2}$ in Theorem 3.1, we get the results of [4].

\section{Competing interests}

The authors declare that they have no competing interests.

\section{Authors' contributions}

This paper is the result of joint work of all authors who contributed equally to the final version of this paper. All authors read and approved the final manuscript.

\section{Author details}

'Department of Electrical Engineering, Northwest A\&F University, Yangling, Shaanxi 712100, China. ${ }^{2}$ Department of Construction and Information Engineering, Guangxi Modern Vocational Technology College, Hechi, Guangxi 547000, China. ${ }^{3}$ School of Civil Engineering, Hebei University of Technology, Tianjin, 300130, China. ${ }^{4}$ Institute of Information Technology, Guilin University of Electronic Technology, Guilin, Guangxi 541004, China.

\section{Acknowledgements}

The authors would like to thank the editors and the referees for their valuable suggestions to improve the quality of this paper. The first author was supported by the scientific research foundation of National Natural Science Foundation (51109180), the National Science \& Technology Supporting Plan from the Ministry of Science \& Technology of P.R. China (2011BAD29B08), the '111' Project from the Ministry of Education of P.R. China and the State Administration of Foreign Experts Affairs of P.R. China (B12007), Fundamental Research Funds for the Central Universities (Z109021310) and the scientific research foundation of National Natural Science Foundation (51279167). 
References

1. Hardy, GH, Littlewood, JE, Polya, G: Inequalities. Cambridge University Press, Cambridge (1952)

2. Mitrinovic, DS, Pecaric, JE, Fink, AM: Inequalities Involving Functions and Their Integrals and Derivatives. Kluwer Academic, Boston (1991)

3. Yang, BC, Rassias, TM: On the way of weight coefficient and research for the Hilbert-type inequalities. Math. Inequal. Appl. 6(4), 625-658 (2003)

4. Jiang, W-D, Hua, Y: On improvement of a Hilbert-type inequality. Comput. Math. Appl. 60(3), 629-633 (2010)

5. Yang, BC: The Norm of Operator and Hilbert-Type Inequality. Science Press, Beijing (2008)

6. Kuang, J: Applied Inequalities. Shandong Science Press, Jinan (2003)

10.1186/1029-242X-2013-511

Cite this article as: Chen et al.: On a strengthened Hardy-Hilbert type inequality. Journal of Inequalities and Applications 2013, 2013:511

Submit your manuscript to a SpringerOpen ${ }^{\circ}$ journal and benefit from:

- Convenient online submission

- Rigorous peer review

- Immediate publication on acceptance

- Open access: articles freely available online

- High visibility within the field

- Retaining the copyright to your article 